\title{
Stereospecific Polymerization of Acrylonitrile Using Acrylonitrile-Urea Canal Complex Initiated by $\gamma$-Ray Irradiation: Roles of Radical Chain Transfer Reagents
}

\author{
Hitoshi Yamazaki, Yukio MiYaZaKi, and Kenji Kamide* \\ Fundamental Research Laboratory of Fibers and Fiber-Forming Polymers, \\ Asahi Chemical Industry Co., Ltd., 11-7 Hacchonawate, \\ Takatsuki, Osaka 569, Japan
}

(Received September 10, 1990)

\begin{abstract}
An attempt was made to clarify the effect of polymerization conditions (e.g., the content of chain transfer reagent, irradiation dose, acrylonitrile (AN)-urea ratio, aging time) on the stereoregularity, the viscosity-average molecular weight $M_{v}$, and the conversion in the radiation-induced polymerization of $\mathrm{AN}$-urea canal complex in the presence of radical chain transfer reagent including alkyl mercaptans, alkylamines, alkyl alcohols and chloroform. The addition of $n$-butyl mercaptan ( $n$-BM) to AN-urea system slowed down the rate of the canal complex formation and decreased the amount of the canal complexes formed. The role of $n$-BM on the formation of AN-urea canal complex was also examined by DSC. The enthalpy of fusion $(\Delta H)$ of AN solid in AN- $n$-BM-urea system decreased with elapse of aging time, although $\Delta H$ of $n$-BM was almost constant in the whole range of aging time. From these findings, it was concluded that $n$-BM is not included in the AN-urea canal complex. The conversion decreased remarkably with an increase in $n$-BM content. When 2 mol\%/AN of $n$-BM was added to AN, $M_{v}$ was almost constant ( $c a$. $M_{v}=2.0 \times 10^{5}$ ), irrespective of the amount of $n$-BM added. Addition of $n$-BM to AN-urea system brings about a small, but significant increase in the content of isotactic triad ( $\mathrm{mm}$ ) by 5 to $14 \%$.
\end{abstract}

KEY WORDS Tacticity / Polyacrylonitrile / $\gamma$-Ray / DSC / Chain Transfer

Reagent / Acrylonitrile-Urea Canal Complex /

As early as 1956 Clasen $^{1}$ first found that 2,3-dimethylbutadiene included in thiourea canal can be polymerized. Unfortunately he examined neither tacticity nor molecular weight of the polymer obtained. Brown and White ${ }^{2}$ proposed in the study of disubstituted butadiene-thiourea complex the concept of selective and stereospecific polymerization of monomer trapped in a canal of thiourea. However, they demonstrated that acrylonitrile (AN) did not form a canal complex with thiourea. White ${ }^{3}$ carried out experiments of irradiation with a beam from X-ray type resonant transformer on $\mathrm{AN}$-urea canal

\footnotetext{
* To whom all correspondence should be addressed.
}

complex at $195 \mathrm{~K}$. He obtained polyacrylonitrile (PAN), whose infra-red spectrum (IR) was shown to be similar with commercially available PAN, although minor difference in the spectroscopy was noted without further characterization of stereoregularity of the polymer.

Kawasaki et al. ${ }^{4}$ examined, using differential thermal analysis (DTA), the condition of formation of $\mathrm{AN}$-urea canal complex, disclosing that the adducts formed at $195 \mathrm{~K}$ were stable below $225 \mathrm{~K}$, above which the adducts decomposed. They also examined polymerization kinetics such as the conversion-dose 
rate-irradiation time relations and qualitative solubility nature of the polymer against popular solvents, including $N, N$-dimethylformamide (DMF), dimethyl sulfoxide (DMSO), $50 \mathrm{wt} \%$ potassium thiocyanate (KSCN) aq. solution, 50 and $70 \mathrm{wt} \%$ zinc chloride $\left(\mathrm{ZnCl}_{2}\right)$ aq. solution, and sulfuric acid. In addition, the absence of ketenimine structure and existence of conjugated $(-\mathrm{C}=\mathrm{N})_{n}$ groups in the PAN were shown by IR analysis. They described that PAN thus prepared has high crystallinity, but gave no information on the stereoregularity of PAN, obtained by $\gamma$-ray irradiation on AN-urea canal complex, which is hereafter simply referred to as $\gamma$-PAN. Hedvig et al. ${ }^{5}$ prepared PAN by $\gamma$-ray radiation polymerization in urea canals, confirming that $\gamma$-PAN had an identical chemical structure with bulkpolymer (by IR method) and was not amorphous (by X-ray diffraction method).

Yoshii and his co-workers ${ }^{6}$ estimated the molar ratio of $\mathrm{AN}$-urea canal complex to be about $1: 1$ by DTA and X-ray diffraction analysis and studied the polymerization of AN at elevated temperatures higher than $77 \mathrm{~K}$ after $\gamma$-ray irradiation on the complex at $77 \mathrm{~K}$ (i.e., after-effect polymerization). They are probably the first who measured the average molecular weights of $\gamma$-PAN, by gel permeation chromatography (GPC), although the physical meaning of their molecular weights were obscure because of oversimplified usage of polystyrene standards for calibration in GPC, in which DMF was used as solvent at $343 \mathrm{~K}$. Later, Yoshii et al. ${ }^{7}$ demonstrated using broad-line nuclear magnetic resonance (NMR) spectroscopy that radicals were generated mainly in $\mathrm{AN}$ in the urea canal complex and initiated the polymerization at $c a .173 \mathrm{~K}$, which was related with the mobility of AN monomer. They also investigated the effect of preparing conditions on the polymer yield, estimating qualitatively by high resolution proton $\left({ }^{1} \mathrm{H}\right)$ NMR the stereoregularity of two $\gamma$-PAN samples. More quantitative analysis of the stereoregularity of $\gamma$-PAN by ${ }^{1} \mathrm{H}$ NMR was energetically undertaken in $1960 \mathrm{~s}$ by Yoshino, $^{9}$ and Matsuzaki et al. ${ }^{10-12}$ : Yoshino gave an assignment of methylene proton peaks of PAN from deuterated AN ( $\alpha-d_{1}$ - and $\alpha, \beta-d_{2}$-polyacrylonitrile). Matsuzaki and his coworkers evaluated diad content of PAN using $\alpha-d_{1}$ $\mathrm{AN}$ and its dependence on polymerization temperature in radical polymerization, and they determined triad and pentad tacticity of PAN based on methine proton peaks in ${ }^{1} \mathrm{H}$ NMR spectra of the polymer from $\beta, \beta-d_{2}-\mathrm{AN}$.

Inoue et al. ${ }^{13,14}$ and Kamide and his collaborators ${ }^{15,16}$ attempted more detailed and comprehensive studies on the stereoregularity of $\gamma$-PAN using ${ }^{13} \mathrm{C}$ NMR: The former paid keen attention to the establishment of the full assignment, with the aid of 2,4-dicyanopentane, of ${ }^{13} \mathrm{C}$ NMR spectrum for $\gamma$-PAN with comparison of chemical shifts theoretically calculated by $\mathrm{CNDO} / 2$ method for determination of triad tacticity. Kamide et al. ascertained the validity of the assignment of iso ( $m m, m$ denotes meso configuration), hetero ( $m r, r$ denotes racemo configuration) and syndio ( $r r)$ signals in ${ }^{13} \mathrm{C}$ NMR spectra by considering the electro-magnetical influence on $\mathrm{CN}$ carbons induced by the interaction of adjacent $\mathrm{CN}$ groups arranged in meso configuration and gave a method for evaluating the pentad tacticity by analyzing the splitting of the CN carbon NMR peak with high magnetic field ${ }^{13} \mathrm{C}$ NMR. In addition, Kamide et al. $^{17}$ determined a Flory theta solvent for $\gamma$-PAN with the content of a $\mathrm{mmmm}$ sequence of $0.37 \pm 0.02$ to be $56.5 \mathrm{wt} \%$ aq. nitric acid at $298 \mathrm{~K}$, by extrapolation of the second virial coefficient $A_{2}$ to zero and they disclosed that unexpectedly larger conformation parameters for $\gamma$-PAN and PAN, prepared by conventional redox polymerization (R-PAN) could be interpreted by considering the rigidity of the chain due to intra-interactions between neighbouring $\mathrm{CN}$ groups in meso sequence.

Now, we have considerable accumulation of scientific knowledge on the polymerization kinetics of $\mathrm{AN}$ by $\gamma$-ray irradiation on $\mathrm{AN}$ - 
urea canal complex and some established techniques for characterizing polymer stereoregularity and other molecular characteristics, but there remains even now a large, unbriged gap between the preparative conditions and polymer characteristics. This motivated the present study.

In this article, we intent to investigate in a very systematic manner the effect of the preparing conditions including the radical transfer reagent, mixing ratio of $\mathrm{AN}$-urea, time of inclusion, irradiation dose, on the rate of polymerization, yield, stereoregularity and viscosity-average molecular weight $M_{v}$ of the polymers and to establish a method of production for controlling the stereoregularity and the average molecular weight of PAN and to present a more detailed mechanism of $\gamma$-ray irradiation-induced polymerization of $\mathrm{AN}$ in urea canal complex.

\section{EXPERIMENTAL}

\section{AN-Urea Canal Complex Formation}

Reagent grade urea manufactured by Wako Chemical Co. (Osaka, Japan) was purified by recrystallization from methanol-water $(5: 1$, v/v at 298 K) mixture. Precipitated urea crystals were filtered, washed with acetone, dried in air and then in vacuo. Reagent grade AN manufactured by Kishida Chemical Co., (Osaka, Japan) was distilled immediately before use. Isopropyl mercaptan, $n$-butyl mercaptan ( $n$ BM), $t$-butyl mercaptan, cyclohexyl mercaptan, thiophenol, $n$-butylamine, triethylamine, $n$-butyl alcohol, $t$-butyl alcohol, isoamyl alcohol, and chloroform were used for chain transfer reagents as received (spectra grade).

$\mathrm{AN}$, to which a given amount of chain transfer reagents was, if necessary, added in advance, was mixed at room temperature in a polyethylene bag with purified urea crystals to give a mixture with a given composition. The AN-urea mixture was stood at $195 \mathrm{~K}$ for a given period of time ( 7 days when not noted specially) to allow complete formation of
AN-urea canal complex.

$\mathrm{AN}$, added with $n$-BM, was mixed with urea to determine from analysis on a differential scanning calorimeter (DSC) thermogram (i.e., exothermic peaks of AN and the canal complex) the rate of the complex formation.

\section{$\gamma$-Ray Induced Polymerization}

Keeping the temperature at $195 \mathrm{~K}$ the complex thus prepared was transferred to a stainless steel Dewar bottle, to which $\gamma$-rays from the radiation source of 14000 curies of cobalt 60, installed in Osaka Prefectural Radiation Research Institute (now, Research Institute, Osaka Prefectural University) were irradiated at $195 \mathrm{~K}$ at a given dose rate for a given time with total doses up to $1.3 \times 10^{6}$ roentgen (R). After a given period of polymerization, a hard solid of polymer-urea mixture was crushed by mixer with distilled water and with which urea contaminated was extracted. The residue was poured into distilled water, washed repeatedly with distilled water and methanol, dipped in methanol overnight, washed again with methanol until complete removal of the unreacted monomers, urea and chain transfer reagent was confirmed. Finally, the polymer was dried in vacuo and used for further analysis.

\section{Tacticity}

The spectra of $\gamma$-PAN samples were measured in the form of $5-10 \mathrm{wt} \%$ solutions in a mixture $(1: 2, \mathrm{v} / \mathrm{v}$ at $298 \mathrm{~K})$ of dimethyl sulfoxide (DMSO) and deuterated DMSO (DMSO- $d_{6}$ ) at $353 \mathrm{~K}$, or in a mixture of $70 \mathrm{wt} \%$ nitric acid in $\mathrm{H}_{2} \mathrm{O}$ and $65 \mathrm{wt} \%$ nitric acid- $d_{1}$ in $\mathrm{D}_{2} \mathrm{O}(1: 2, \mathrm{v} / \mathrm{v}$ at $298 \mathrm{~K})$ at $300 \mathrm{~K} .{ }^{13} \mathrm{C} \mathrm{NMR}$ spectra were measured using the spectrometer JEOL FX-200 (50.15 MHz).

Triad isotacticity $(\mathrm{mm})$ based on the integrated peak intensity ratio was determined according to the method proposed in the previous paper. ${ }^{16}$ 
Solution Viscosity and Viscosity-Average Molecular Weight

The solution viscosity was measured for solutions in aq. $67 \mathrm{wt} \% \mathrm{HNO}_{3}$ using a modified dilution-type Ubbelohde viscometer at $298 \pm$ $0.01 \mathrm{~K}$. The limiting viscosity number $[\eta]$ was determined for each sample by Huggins plots.

Viscosity-average molecular weight $\left(M_{v}\right)$ was evaluated from $[\eta]$ through use of the Mark-Houwink-Sakurada (MHS) equation

$$
[\eta]=0.217 \times M_{w}^{0.57}
$$

in aq. $67 \mathrm{wt} \% \mathrm{HNO}_{3}$ at $298 \mathrm{~K}$

established by Kamide et al. ${ }^{17}$ for $\gamma$-PAN. Here $M_{w}$ is the weight-average molecular weight and the possible difference in the parameters in MHS equation between $\gamma$-PAN samples having different tacticity was neglected.

\section{Differential Scanning Calorimetry (DSC)}

DSC measurements were performed with a DSC model 200 (Seiko Electronics Co., Tokyo) at a constant flow rate of $\mathrm{N}_{2}$ gas $\left(100 \mathrm{~cm}^{3}\right.$ $\left.\mathrm{min}^{-1}\right)$ in the range of temperature from 123 to $273 \mathrm{~K}$. Given amounts of AN, urea and if necessary, $n$-BM as chain transfer reagent were sealed into an aluminum $(99.0 \%$ purity) cylinder disc-type vessel, which was in advance maintained at $195 \mathrm{~K}$, and transfered quickly to the sample holder of the DSC apparatus, preliminarily thermoregulated at a temperature below $123 \mathrm{~K}$ and heated at a rate of $3 \mathrm{~K} \mathrm{~min}^{-1}$. In this case, reagent grade urea was recrystallized in methanol-water $(5: 1, \mathrm{v} / \mathrm{v}$, $293 \mathrm{~K}$ ) mixture, dried, and powdered by mortar. An aluminum vessel containing the same amount of urea as that in the sample vessel was utilized as reference. Here, we define the time consumed for formation of $\mathrm{AN}$-urea canal complex at $195 \mathrm{~K}$ as aging time. From an experimental view point, both the time necessary for the base line to attain the steady state and measuring time were excluded for the sake of simplicity in counting the aging time. From DSC thermogram the enthalpy changes $\Delta H$ in melting of $\mathrm{AN}$-urea canal complex, $\mathrm{AN}$ and urea crystals were evaluated as a function of aging time.

\section{Electron Spin Resonance (ESR)}

ESR spectra were measured at $77 \mathrm{~K}$ in liquid nitrogen on a JEOL 3BX X-band spectrometer (microwave frequency, 9188.8 MHz) under the following conditions: magnetic field modulation, $100 \mathrm{KHz}$; width of modulation, 2 gauss; $g$-value was calculated from ${ }^{1} \mathrm{H}$ NMR frequency. Sample tube with outer radius of $4.7 \mathrm{~mm}$ was consisted of two parts: lower quartz part with $10 \mathrm{~cm}$ length and upper pyrex glass part with $20 \mathrm{~cm}$ length.

Given amounts of finely powdered pure urea, distilled $\mathrm{AN}$ and reagent grade $n$-BM (if necessary) were put into the tube. Its pyrex glass part was sealed keeping lower quartz part under $10^{-3}$ torr at $77 \mathrm{~K}$. After maintaining at $195 \mathrm{~K}$ (dry ice) for 4 days, the sealed tube was irradiated with $\gamma$-ray at dose rate of $3 \times 10^{5}$ $\mathrm{R} \mathrm{h}^{-1}$ for $3 \mathrm{~h}$ at $195 \mathrm{~K}$. After the irradiation was completed, the sample tube was transfered and immersed into liquid nitrogen in dewar bottle for storage until ESR measurements. ${ }^{18}$

\section{X-Ray Diffraction}

X-Ray diffraction patterns of AN-urea $(1: 1.5, \mathrm{~mol} / \mathrm{mol})$ mixture aged for 7 days at $195 \mathrm{~K}$ were recorded under reduced pressure $\left(\sim 10^{-3}\right.$ Torr $)$ in a X-ray diffractometer RU-300PL (acceleration voltage, $58 \mathrm{kV}$; electron beam current, $280 \mathrm{~mA}$ ) (Rigaku Denki, Tokyo), equipped with thermoregulator apparatus (temperature control range; $77-293 \mathrm{~K}$ ). Copper $K_{\alpha}$ radiation, monochromatized by an $\mathrm{Ni}$ filter, was used.

\section{RESULTS AND DISCUSSION}

Figure 1 shows a plot of the conversion (a), $M_{v}$ (b) and the content (mol\%) of isotactic triad $\mathrm{mm}$ (c) of the polymer as a function of the total dose of $\gamma$-ray irradiation. The conversion increases with total dose, approaching an asymptotic value $(80 \%)$ at $7 \times 10^{5} \mathrm{R}$. 


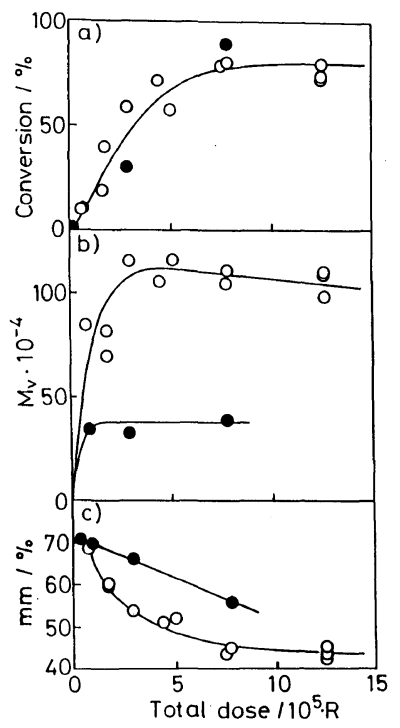

Figure 1. Effect of irradiation total dose on conversion (a), $M_{v}$ (b) and $\mathrm{mm}$ content (c) of the PANs obtained in AN-urea canal complex: $\bigcirc, A N-u r e a=1: 1.5(\mathrm{~mol} / \mathrm{mol})$; - $\mathrm{AN}-$ urea $=1: 3.0(\mathrm{~mol} / \mathrm{mol})$.

Variation of the molar ratio of AN to urea in the range from 1.5 to 3.0 yields only insignificant effect on the conversion-total dose relation.

$M_{v}$ increases rapidly with an increase in total dose, and gradually decreases after passing through maximum (when $\mathrm{AN}-\mathrm{urea}=1.0: 1.5$, $\mathrm{mol} / \mathrm{mol}$ ) or approaches an asymptotic value (when AN-urea =1.0:3.0, $\mathrm{mol} / \mathrm{mol}$ ). The total dose giving the maximum or asymptotic $M_{v}$ seems smaller for the system of smaller $\mathrm{AN} /$ urea ratio. The ratio $\mathrm{AN} /$ urea is expected to be an important factor governing the maximum or asymptotic value of $M_{v}$ : Larger AN/urea ratio yields larger $M_{v}$ at constant total dose. This experimental fact suggests strongly that larger molar ratio of urea to $\mathrm{AN}$ brings about numerous smaller canal complexes in size.

The isotacticity of the polymer is higher when the polymer is formed at smaller $\gamma$-ray dose. At a given total dose, the isotacticity becomes higher for lower AN/urea ratio (in other words, when the larger amount of urea is added to
AN). It is reasonable to consider that $\mathrm{AN}$ monomer is included in a more regular manner in the $\mathrm{AN}$-urea mixture with larger urea composition, and the canal complex with higher regularity has lower activation energy of initiation reaction of polymerization. ${ }^{4}$ Therefore, at relatively earlier stage AN monomers in the complex with higher regularity are, more or less, preferentially polymerized, yielding PAN with higher stereoregularity. Further irradiation of $\gamma$-ray polymerizes $\mathrm{AN}$ in the $\mathrm{AN}$-urea canal complex with lower regularity, resulting in PAN with less stereoregularity.

In order to synthesize highly stereoregular PAN with high yield, an increase in the portion of the AN-urea canal complex with high regularity might be an alternative and useful method, which has not, unfortunately, been experimentally realized until now. Provided that (1) $\gamma$-ray irradiation polymerization of AN-urea canal complex obeys a radical polymerization mechanism and that (2) urea makes exclusively the canal complex with $\mathrm{AN}$ and does not form any complex with a chain transfer reagent, and that (3) the reagent exists only at exterior of the AN-urea canal complex, or at most the complex with high irregularity. Thus the chain transfer reagent is expected to suppress the polymerization of $\mathrm{AN}$ located at the end of the regular canal complex and/or $\mathrm{AN}$ in the less regular complex, allowing the polymerization of $\mathrm{AN}$ alone in the regular canal complex, which gives the highly stereoregular PAN. In addition, the addition of chain transfer reagents may be effective in controlling the molecular weight of PAN. Therefore, an attempt was made to carry out the $\gamma$-ray irradiation polymerization of $\mathrm{AN}$-urea canal complex under the coexistence of radical chain transfer reagents and verify the effectiveness of controlling $M_{v}$ of the polymer. The results are summarized in Table I.

Figure 2 shows the correlations between the isotactic triad content and $M_{v}$ of $\gamma$-PAN prepared using chain transfer reagents. In the 
Table I. Effects of radical chain transfer agents on polymerization of AN-urea canal complex at $195 \mathrm{~K}^{\mathbf{a}}$

\begin{tabular}{|c|c|c|c|c|c|c|}
\hline \multirow{2}{*}{ Radical transfer agents } & \multirow{2}{*}{ Yield $/ \%$} & \multirow{2}{*}{$M_{v}^{\mathrm{b}} / 10^{4}$} & \multicolumn{3}{|c|}{ Triad tacticity } & \multirow{2}{*}{$\begin{array}{c}\text { Transfer const. } \\
\left(60^{\circ} \mathrm{C}\right)\end{array}$} \\
\hline & & & $I$ & $H$ & $S$ & \\
\hline none & 58.0 & 115.4 & 52.1 & 34.2 & 13.6 & \\
\hline Isopropyl mercaptan & 41.1 & 9.0 & 61.9 & 26.6 & 11.5 & \\
\hline$n$-Butyl mercaptan & 43.5 & 18.2 & 63.5 & 26.3 & 10.2 & $(0.66--25)^{d}$ \\
\hline$t$-Butyl mercaptan & 47.7 & 23.1 & 57.3 & 31.9 & 10.8 & \\
\hline Cyclohexyl mercaptan. & 41.4 & 26.8 & 64.8 & 26.2 & 9.0 & \\
\hline Thiophenol & 39.6 & 18.5 & 66.4 & 24.6 & 9.0 & \\
\hline$n$-Butylamine & 23.0 & 28.4 & 53.9 & 34.0 & 12.1 & \\
\hline Triethylamine & 23.7 & 44.2 & 53.0 & 31.7 & 15.3 & $0.17-0.66$ \\
\hline$n$-Butyl alcohol & 52.0 & 108.1 & 50.1 & 35.3 & 14.4 & $154.2 \times 10^{-5}$ \\
\hline$t$-Butyl alcohol & 53.3 & 110.3 & 50.7 & 36.4 & 12.9 & $4.4 \times 10^{-5}$ \\
\hline Isoamyl alcohol & 64.4 & 61.3 & 52.7 & 36.0 & 11.3 & \\
\hline Chloroform & 51.6 & 94.7 & 52.8 & 34.8 & 12.4 & $56.4 \times 10^{-5}$ \\
\hline
\end{tabular}

a Dose rate, $1.0 \times 10^{5} \mathrm{R} \mathrm{h}^{-1}$ in air; Irrad. time, $5 \mathrm{~h}$; AN-Urea, $1.0: 1.5 \mathrm{~mol} / \mathrm{mol}$.

b Viscosity average molecular weight.

c Reference 20.

d Estimated on the polymerization of styrene, or methyl methacrylate monomer.

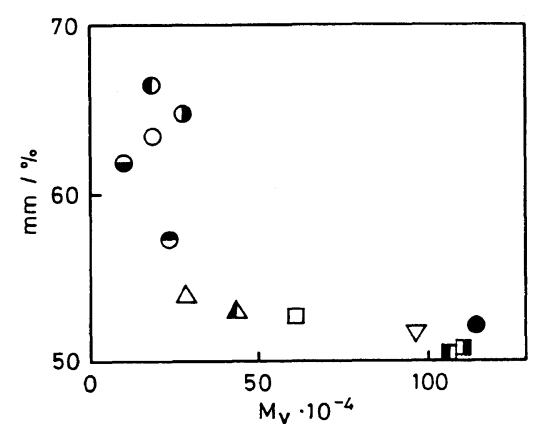

Figure 2. Relations between viscosity average molecular weight $M_{v}$ and isotactic triad content of the polyacrylonitriles obtained in urea canal complex containing $1 \mathrm{~mol} \% / \mathrm{AN}$ transfer agent: irradiation dose rate, $1.0 \times 10^{5}$ $\mathrm{R} \mathrm{h}^{-1}$; irradiation time, $5 \mathrm{~h} ; \mathrm{AN}-$ urea $=1.0: 1.5(\mathrm{~mol} / \mathrm{mol})$; - without transfer agent; $\bigcirc$, n-butyl mercaptan; $\odot$, isopropyl mercaptan; $\mathbf{O}$, cyclohexyl mercaptan; $\mathbf{D}$, thiophenol; $\Theta, t$-butyl mercaptan; $\triangle, n$-butyl amine; $\Delta$, triethylamine; $\nabla$, chloroform; $\square$, isoamyl alcohol; $\square$, $n$-butyl alcohol; $\mathbf{\square}, t$-butyl alcohol.

figure filled circle corresponds to the case where no transfer reagents is added (as reference). The addition of mercaptans to $\mathrm{AN}$-urea system brings about a small, but significant increase in the content of isotactic triad by 5 to $14 \%$ and a drastic decrease in $M_{v}$ (i.e.,
1/13-1/4 of $M_{v}$ of PAN prepared without chain transfer reagent).

The crystal transition is known to be accompanied by the formation of $\mathrm{AN}$-urea canal complex. When urea, whose crystal form is tetragonal, is mixed well with AN liquid and settled at sufficiently low temperature, urea crystal changes from tetragonal to hexagonal, yielding a canal complex with AN. ${ }^{19}$ Finally we obtain a hexagonal pillow of the compiex with diameter of $5.2 \AA$. It is also well known ${ }^{19}$ that linear paraffine derivatives are included, but the branched molecules with bulky side chains and cyclic molecules are not included in the canal complex. It was experimentally confirmed from Table I that the addition to AN-urea system of not only bulky molecules, such as cyclohexyl mercaptan and $t$-butyl mercaptan, but also linear molecules including $n$-alkyl mercaptan induces an increase in isotacticity (i.e., an increase in content of $\mathrm{mm}$ triad) and a decrease in $M_{v}$.

Accordingly, chain transfer reagents are not involved together with AN into canal complex, but may be dispersed between canal complexes, receiving a propagating radical of $\mathrm{AN}$ 
monomer at the end of the complex and resulting in termination of further polymerization reaction of $\mathrm{AN}$ monomer existing in less regular complexes. This termination can explain reasonably the following experimental findings: The addition of chain transfer regent brings about, more or less, the suppression of the formation of less stereoregular PAN and decrease of the average molecular weight of PAN. Interestingly amines are effective in lowering $M_{v}$ only and ineffective in increasing isotacticity. Therefore, it is considered that amines retard the formation of $\mathrm{AN}$-urea canal complex and lower the regularity of the complex, probably by invasion of amine molecules into the interior of the complex. Alcohols and chloroform, whose chain transfer coefficients are usually low, ${ }^{20}$ have only smaller effect on increase in isotacticity and a lowering of $M_{v}$.

In order to confirm, directly by experiment, the above speculation on the role of a chain transfer reagent in $\mathrm{AN}$-urea canal complex formation, the DSC technique was applied to these systems.

Figure 3 shows a DSC thermogram of a mixture of AN, $n$-butyl mercaptan ( $n$-BM) and urea $(1.0: 0.1: 3.0$, molar ratio), measured after being maintained for $24 \mathrm{~h}$ at $195 \mathrm{~K}$. Melting points of $n$-BM, AN and AN-urea canal complex each were estimated in preliminary experiments to be $155.0 \mathrm{~K}, 184.1 \mathrm{~K}$, and $233.8 \mathrm{~K}$, respectively. These coincide well with the temperatures of peaks $a, b, c$ in the figure,

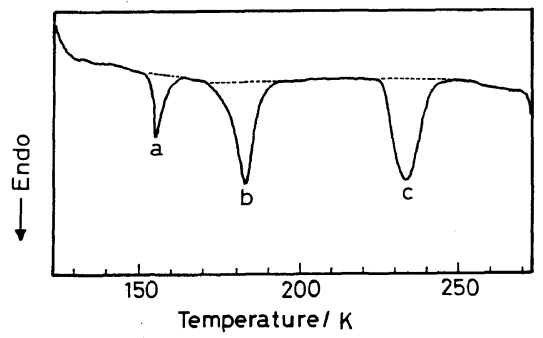

Figure 3. DSC curve of AN- $n$-BM-urea canal complex: $\mathrm{A}$ N- $n$-BM-urea $=1: 0.1: 3$ (molar ratio); a, $n$-BM; b, AN; c, canal complex. respectively. It was observed that the peak temperature of peak $\mathrm{c}$ was always constant $(233.8 \mathrm{~K})$, independent of the amount of $n$-BM coexisting in the $\mathrm{AN}$-urea mixture. This is strong indication that $\mathrm{AN}$-urea canal complex does not contaminate any $n$-BM. The enthalpy of fusion of the AN-urea complex $\Delta H$, estimated from the area under peak c, is apparently proportional to the amount of AN-urea canal complex formed. The relative amounts of free $\mathrm{AN}$ and $n$-BM are also estimated from the areas under peaks $b$ and $a$ $(\Delta H)$, respectively.

Figure 4a shows $\Delta H$ of canal complex in $\mathrm{AN}$-urea and $\mathrm{AN}-n$-BM-urea systems as a function of aging time. Coexistence of $n$-BM retards significantly the formation of $\mathrm{AN}$-urea canal complex. Figure $4 \mathrm{~b}$ shows plots of the relative amounts of free $\mathrm{AN}$ and $n$-BM, expressed by $\Delta H$, versus aging time. The amount of free $n$-BM in $\mathrm{AN}-n-\mathrm{BM}-\mathrm{urea}=$

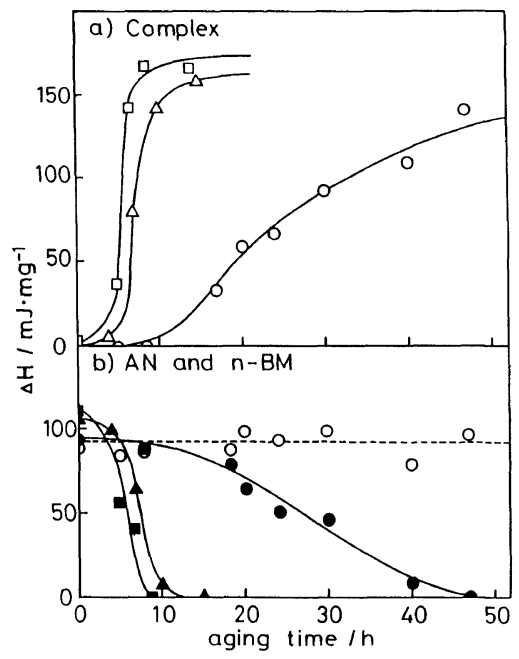

Figure 4. Aging time dependence of $\Delta H$ of canal complex in $\mathrm{AN}$-urea and $\mathrm{AN}-n$-BM-urea systems (a) and $\Delta H$ of free $\mathrm{AN}$ in $\mathrm{AN}$-urea and $\mathrm{AN}-n-\mathrm{BM}$-urea systems and free $n$-BM in AN- $n$-BM-urea system (b): a) $\square, \mathrm{AN}$-urea $=$ $1.0: 3.0$ (molar ratio); $\triangle, \mathrm{AN}-n-\mathrm{BM}$-urea $=1.0: 0.01: 3.0$; $\bigcirc, \mathrm{AN}-n-\mathrm{BM}-\mathrm{urea}=1.0: 0.1: 3.0$. b) $\square$, free $\mathrm{AN}$ in $\mathrm{AN}-$ urea $=1.0: 3.0 ; \boldsymbol{\Delta}$, free $\mathrm{AN}$ in $\mathrm{AN}-n-\mathrm{BM}-$ urea $=$ $1.0: 0.01: 3.0 ; 0$, free $\mathrm{AN}$ in $\mathrm{AN}-n-\mathrm{BM}-\mathrm{urea}=1.0: 0.1$ : $3.0 ; \bigcirc$, free $n$-BM in $\mathrm{AN}-n-\mathrm{BM}-$ urea $=1.0: 0.1: 3.0$ (broken line). 
$1.0: 0.1: 3.0$ (molar ratio) system, not included in the complex, was maintained constant, irrespective of aging time. This is other experimental evidence indicating that $n$-BM is excluded from the complex. It is interesting to note that at the time when peak b disappears, the area under peak $c$ attains an assymptotic value. In other words, free $\mathrm{AN}$ is completely consumed for formation of the $\mathrm{AN}$-urea canal complex. The breadth of the peak $\mathrm{c}$ reflects, of course, heterogeneity, that is, distributions of regularity (perfectness) and size of the complexes. A kind of fractionation between $\mathrm{AN}$ and $n$-BM from their mixture occurs during the course of the formation of the $\mathrm{AN}$-urea canal complex and as a result the rate of the complex formation becomes slower when a larger amount of chain transfer reagent is added to the system. The above experimental results show that mercaptans have almost the same effect in $\gamma$-ray irradiation polymerization of $\mathrm{AN}$ as that of typical chain transfer reagents in radical polymerization. This also suggests strongly that polymerization of AN by $\gamma$-ray irradiation on $\mathrm{AN}$-urea canal complex is a kind of radical polymerization as was demonstrated by Ohmori et al. ${ }^{21}$ by use of ESR that polymerization of 1,3-butadiene initiated by $\gamma$-ray irradiation on 1,3-butadiene-urea canal complex obeyed radical polymerization mechanism. Using ESR Magat ${ }^{18}$ confirmed that solid polymerization of AN by $\gamma$-ray irradiation was also a radical polymerization. Note that the polymer prepared by Magat is atactic.

Figures $5 \mathrm{a}$ and $5 \mathrm{~b}$ show ESR spectra of AN-urea canal complex and AN, measured at $77 \mathrm{~K}$ immediately after irradiation at $195 \mathrm{~K}$ by $\gamma$-ray. Full assignment of hyper-fine structure of the spectrum for $\gamma$-ray irradiated $\mathrm{AN}$-urea canal complex is impossible due to its complexity, but the spectrum can be assigned to exhibit five lines whose intensity ratios are $1: 4: 6: 4: 1$. When pure $\mathrm{AN}$ is irradiated by $\gamma$-ray at $195 \mathrm{~K}$, no significant spectrum was observed (Figure $5 \mathrm{~b}$ ). This means that in the system of pure AN alone, generation of the radical by $\gamma$-ray irradiation at $195 \mathrm{~K}$ is relatively suppressed as compared with that in $\mathrm{AN}$-urea system. Kimura ${ }^{22}$ pointed out that coexistence of urea has an effect of lowering activation energy of polymerization reaction of $\mathrm{AN}$.

Figures $6 \mathrm{a}, 6 \mathrm{~b}$ and $6 \mathrm{c}$ show the X-ray diffraction patterns of AN solid, urea and unirradiated AN-urea canal complex, respectively, measured at $77 \mathrm{~K}$. By comparing these three patterns we can elucidate peaks due to the complex as shown as shadowed area in the figure.

In order to follow crystallographical change of AN-urea canal complex during the polymerization process we employed $\mathrm{X}$-ray in place of $\gamma$-ray as initiator and $\mathrm{AN}$-urea canal

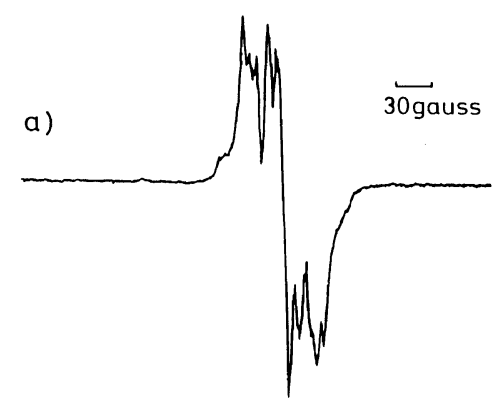

b)

Figure 5. ESR spectra of AN-urea canal complex (a) and AN (b) after $\gamma$-ray irradiation: irradiation dose, $0.9 \times 10^{6} \mathrm{R}$; irradiation temperature, $195 \mathrm{~K}$; measuring temperature, $77 \mathrm{~K}$; a, AN-urea =1:1.5 (molar ratio); b, AN solid.

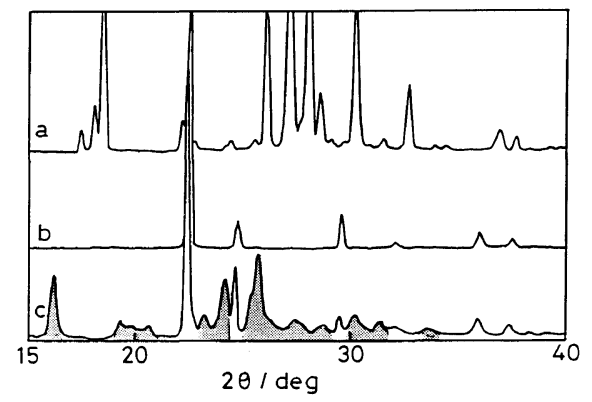

Figure 6. X-Ray diffraction patterns of AN solid (a), urea (b) and AN-urea canal complex (c) at $77 \mathrm{~K}$. 


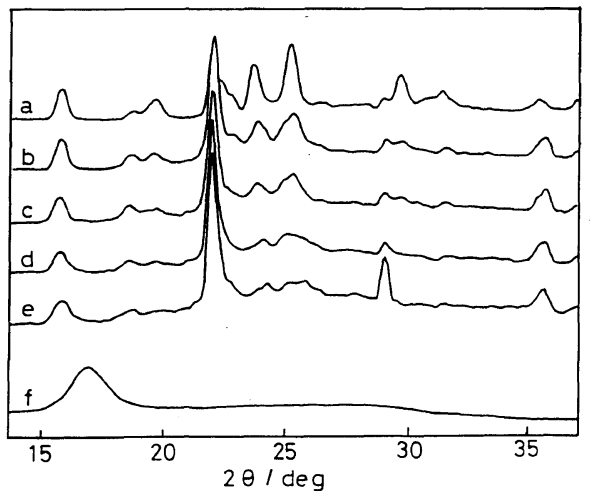

Figure 7. Dependence of X-ray irradiation time on diffraction patterns of $\mathrm{AN}$-urea canal complex irradiated with X-ray at $195 \mathrm{~K}$ : a, $0 \mathrm{~min}$; b, $71 \mathrm{~min}$; c, $101 \mathrm{~min}$; d, $161 \mathrm{~min}$; e, $281 \mathrm{~min}$; f, $\gamma$-PAN powder $(m m=72 \%$, $M_{v}=23 \times 10^{4}$ ), measured at $293 \mathrm{~K}$.

complex was placed in X-ray diffraction apparatus.

Figures $7 \mathrm{a}-\mathrm{e}$ show the $\mathrm{X}$-ray diffraction patterns of $\mathrm{AN}$-urea canal complex, irradiated at $195 \mathrm{~K}$ with $\mathrm{X}$-ray for different period of time (from 0 to $281 \mathrm{~min}$ ). In this case, the measuring time of X-ray diffraction (ca. $20 \mathrm{~min}$ ) was not taken into account of the irradiation time. In Figure $7 \mathrm{f}$ is shown for the sake of comparison the X-ray diffraction pattern of $\gamma$-PAN powder $\left(\mathrm{mm}=72 \%, M_{v}=2.3 \times 10^{5}\right)$. As irradiation period becomes longer a peak at diffraction angle $2 \theta$ of $16.3^{\circ}$ becomes slightly broader and other peaks diminish. The peak at $2 \theta=16.3^{\circ}$ is located near a peak observed in PAN $(290 \mathrm{~K})$, the former can thus be assigned as the diffraction peak of $\mathrm{AN}$ in the canal complex, which is considered to be arranged in a similar manner as that in PAN crystal (in other words, the repetition unit of AN in the canal complex may be almost the same as that in PAN crystal). Polymerization of $\mathrm{AN}$ in the $\mathrm{AN}$-urea canal complex can be initiated by X-rays. Figure 7 shows that as polymerization proceeds the structure of AN-urea canal complex is destroyed gradually. Our present results coincide well with Yoshii et al.'s observation, ${ }^{7}$ by broad-line NMR, on the collapse of canal with proceeding of polymerization, yielding

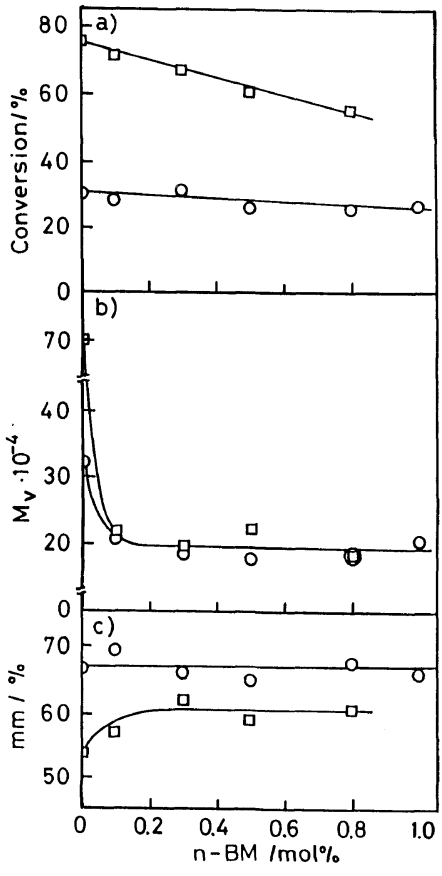

Figure 8. Effects of the amount of $n$-BM added as a chain transfer regent on conversion (a), $M_{v}$ (b) and $m m$ content (c) of PAN obtained in $\gamma$-ray induced polymerization of AN-urea canal complex at $195 \mathrm{~K}$ : irradiation time, $5.0 \mathrm{~h}$; $\mathrm{AN}$-urea $=1: 3$ (molar ratio); irradiation dose rate $\mathrm{O}$, $0.58 \times 10^{5} \mathrm{R} \mathrm{h}^{-1} ; \square, 1.55 \times 10^{5} \mathrm{R} \mathrm{h}^{-1}$.

\section{free $\mathrm{AN}$ monomers.}

Among mercaptans, confirmed to be effective as chain transfer reagents, we chose $n$-BM to investigate the effect of amount of the addition of $n$-BM on the $\gamma$-ray irradiation induced polymerization of $\mathrm{AN}$ in urea canal complexes at two different doses.

Figures 8 and 9 show the experimental results on the conversion, $M_{v}$ and $m m$ content of the polymer. In this case, the AN-urea ratio was taken as $1.0: 3.0(\mathrm{~mol} / \mathrm{mol})$ in Figure 8 and $1.0: 1.5(\mathrm{~mol} / \mathrm{mol})$ in Figure 9. The unfilled circle is the total dose of $0.58 \times 10^{5} \mathrm{R} \mathrm{h}^{-1}$ and unfilled square is that of $1.55 \times 10^{5} \mathrm{R} \mathrm{h}^{-1}$. The polymer yield, expressed by conversion, decreases roughly linearly with increase in the composition of $n$-BM in the mixture. This finding can be quantitatively explained in terms of significant lowering of the formation rate by 


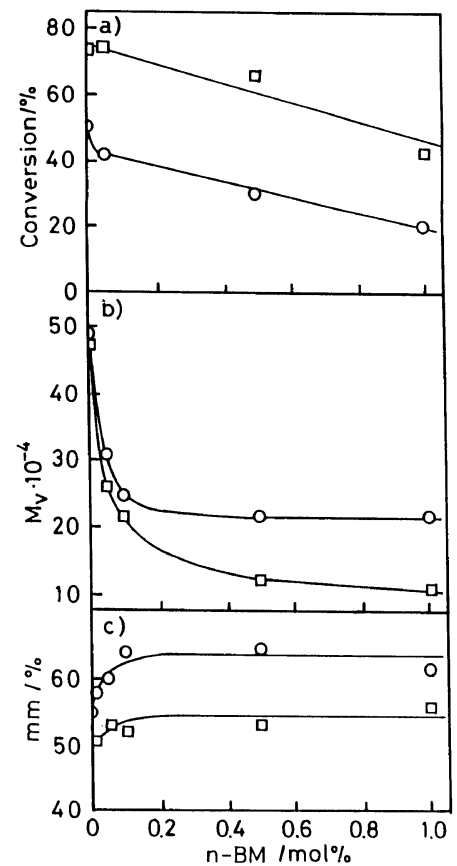

Figure 9. Effects of the amount of $n$-BM added as a chain transfer regent on conversion (a), $M_{v}$ (b) and $m m$ content (c) of PAN obtained in $\gamma$-ray induced polymerization of AN-urea canal complex at $195 \mathrm{~K}$ : irradiation time, $5.0 \mathrm{~h}$; $\mathrm{AN}$-urea $=1.0: 1.5$ (molar ratio); irradiation dose rate $\mathrm{O}$, $0.58 \times 10^{5} \mathrm{R} \mathrm{h}^{-1} ; \square, 1.55 \times 10^{5} \mathrm{R} \mathrm{h}^{-1}$.

increase of the amount of $n$-BM added (see Figure 4). This rate of decreasing is extremely small for a smaller dose rate $\left(0.58 \times 10^{5} \mathrm{R} \mathrm{h}^{-1}\right)$ when $\mathrm{AN}$-urea ratio is $1.0: 3.0(\mathrm{~mol} / \mathrm{mol})$. However, in all other cases, examined here, the rate was kept nearly constant $(\cong 25 \% / n$-BM mol\%), regardless of $\mathrm{AN}-$ urea ratio and dose rate.

In the absence of any chain transfer reagent $M_{v}$ of the polymer synthesized from ANurea $=1.0: 3.0(\mathrm{~mol} / \mathrm{mol})$ depends only on the irradiation dose (see Figure 1b), and the addition of a very small amount of $n$-BM (larger than $0.1 \mathrm{~mol} \%$ of $\mathrm{AN}$ monomer) diminishes $M_{v}$ drastically, which is almost constant (ca. $20 \times 10^{4}$ for AN-urea (1.0:3.0, $\mathrm{mol} / \mathrm{mol}$ ), irrespective of the dose rate and $c a$. $22 \times 10^{4}$ for AN-urea $(1.0: 1.5, \mathrm{~mol} / \mathrm{mol})$ at the dose rate of $\left.0.58 \times 10^{5} \mathrm{R} \mathrm{h}^{-1}\right)$. This indicates that the longitudinal size (along $c$ axis) of AN-urea canal complex is approximately constant regardless of the perfection of the crystal structure. An exception is the case of AN-urea $=1.0: 1.5(\mathrm{~mol} / \mathrm{mol})$ combined with the dose rate of $1.55 \times 10^{5} \mathrm{R} \mathrm{h}^{-1}$, where $M_{v}$ continues to decrease without limit in the range of the $n$-BM content ( $\mathrm{mol} \%$ ) of less than unity.

$m m$ content seems to be predominantly dependent of the dose rate: A lower dose rate yields highly isotactic polymer. The addition of chain transfer reagent by small amount is significant to increase the isotacticity, but further addition beyond $0.1 \mathrm{~mol} \%$ is ineffective in this respect. In other words, a 5-10\% increase in $\mathrm{mm}$ content can be expected by adding a small amount of $n$-BM. The effect of the dose rate on $M_{v}$ and isotacticity of polymer is not practically influenced by addition of chain transfer reagent.

Lower molar ratios of urea to $\mathrm{AN}$ bring about less isotactic polymer, implying that less regular AN-urea canal complexes may be formed due to lower urea ratio. If this speculation is true, a larger molar ratio of urea to AN will yield the polymer with higher isotacticity. In addition, an insignificant effect, on the isotacticity of $\gamma$-PAN, of addition of large amount of radical chain transfer reagent to AN-urea mixture (larger than $0.2 \mathrm{~mol} \%$ / $\mathrm{AN}$ ) and an independence of the polymer molecular weight upon the largely excessive addition of chain transfer reagent seem to support the hypothesis proposed in this study that a AN propagating radical in highly regular portion of canal complex is not influenced by the action of a chain transfer reagent.

Figures 10a and 10b show the influences of the urea/AN molar ratio on $\mathrm{mm}$ content (a) and $M_{v}$ (b). As urea/AN ratio increases, the isotacticity of polymer increases and $M_{v}$ decreases. Figure 10 suggests that under the condition of larger urea/AN ratio highly regular complexes with shorter length are formed.

Figures $11 \mathrm{a}-\mathrm{c}$ show the change in conver- 


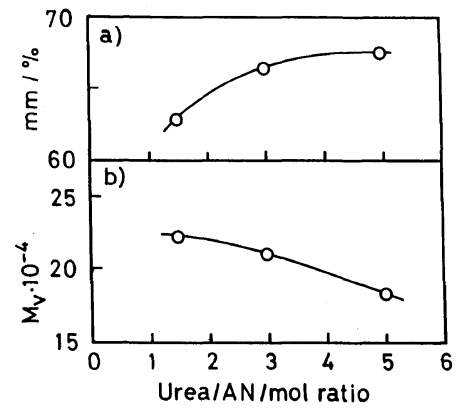

Figure 10. Influence of urea-AN molar ratio on $\mathrm{mm}$ content (a) and $M_{v}$ (b): urea-AN canal complex containing $1 \mathrm{~mol} \% n-\mathrm{BM} / \mathrm{AN}$; irradiation dose, $2.9 \times 10^{5} \mathrm{R}$.

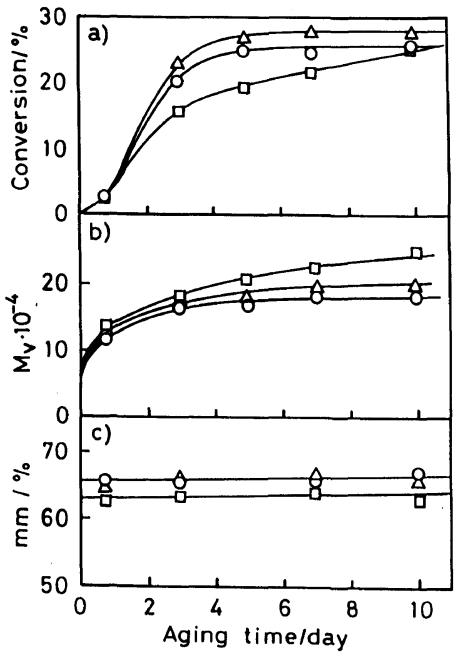

Figure 11. Influence of aging time on conversion (a), $M_{v}$ (b) and $m m$ content (c) for $\mathrm{AN}-n$-BM-urea systems: irradiation dose rate, $0.58 \times 10^{5} \mathrm{R} \mathrm{h}^{-1}$; irradiation time, $5.0 \mathrm{~h} ; \mathrm{AN}-n$-BM-urea (molar ratio) $0,1.0: 0.01: 5.0 ; \triangle$, $1.0: 0.01: 3.0 ; \square, 1.0: 0.01: 1.5$.

sion, $M_{v}$ and $\mathrm{mm}$ content of polymer with aging time (i.e., time allowed for the complex formation) for $\mathrm{AN}-n$-BM-urea (1.0:0.01: 1.5-3.0, molar ratio) systems. The conversion increases with aging time, approaching an asymptotic value, which lies between 25 and $30 \%$. The time of attainment of asymptotic value depends on the relative ratio of $\mathrm{AN}$-urea: Longer time is needed at larger $\mathrm{AN} /$ urea ratio. For example, at AN-urea $=1.0: 1.5(\mathrm{~mol} / \mathrm{mol})$ the time is estimated to be roughly longer than 10 days.

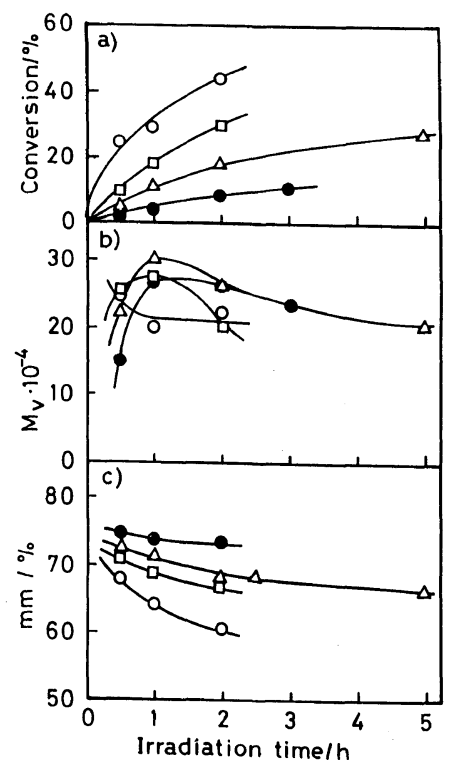

Figure 12. Effects of the irradiation time on conversion (a), $M_{v}$ (b) and $m m$ content (c) for $\mathrm{AN}-n$-BM-urea system: AN- $n$-BM-urea $=1.0: 0.01: 3.0$ (molar ratio); irradiation dose rate $0,0.3 \times 10^{5} \mathrm{Rh}^{-1} ; \triangle, 0.58 \times 10^{5} \mathrm{Rh}^{-1} ; \square$, $1.0 \times 10^{5} \mathrm{Rh}^{-1} ; \mathrm{O}, 3.0 \times 10^{5} \mathrm{Rh}^{-1}$.

$M_{v}$ increases rapidly with aging time, approaching an asymptotic value, which is smaller at a smaller $\mathrm{AN} /$ urea ratio. The time of attainment of asymmetric value is shorter also at smaller AN/urea ratio. This implies that at larger urea/AN ratio (under rich urea condition) the size along with $\mathrm{c}$ axis of canal complex becomes shorter and the number of complexes increases. Isotacticity remains almost constant over a wide range of aging time employed and has a tendency to increase slightly with decrease in $\mathrm{AN} /$ urea ratio. This means that the urea canal complexes with the same degree of regularity are formed over a wide range of aging time and higher urea/AN ratio induces us more regular canal complexes as was found in Figure 10.

Figures $12 \mathrm{a}-\mathrm{c}$ show the effect of the irradiation time on the conversion, $M_{v}$ and isotacticity of the polymer from $\mathrm{AN}-n$ BM-urea (1.0: $0.01: 3.0$, molar ratio) system. The number on the curve denotes the dose rate 
$\times 10^{-5} \mathrm{R} \mathrm{h}^{-1}$. The conversion gets larger more quickly with irradiation time at a larger dose rate. As irradiation time becomes longer, $M_{v}$ increases, then decreases gradually after passing through a maximum. At a dose rate of $3 \times 10^{5} \mathrm{R} \mathrm{h}^{-1}$ maximum of $M_{v}$ could not be observed experimentally. Yoshii et al. ${ }^{8}$ reported similar behavior for AN-urea system, in which no transfer agent was added. They observed using gel chromatography, in which DMF solution of the polymer with concentration of $0.2 \mathrm{wt} \%$, prepared at $413 \mathrm{~K}$, was flowed at $343 \mathrm{~K}$, that at a later stage of polymerization the molecular weight distribution of the polymer becomes broader, especially in the low molecular weight region. The isotacticity of the polymer prepared by initiating the complex for a given time if the dose rate is smaller, is higher and the tacticity of the polymer, prepared under constant dose rate, decreases monotonically with irradiation time. These results can be reasonably explained by the following hypothesis: At a relatively earlier stage of irradiation $\mathrm{AN}$ in the canal complex with higher regularity is initiated and polymerized and by further irradiation, $\mathrm{AN}$ in the complex with less regularity is initiated. In other words, the activation energy of the initiation reaction is smaller in the former than in the latter.

Figure 13 shows the experimental relations among irradiation dose rate, irradiation time

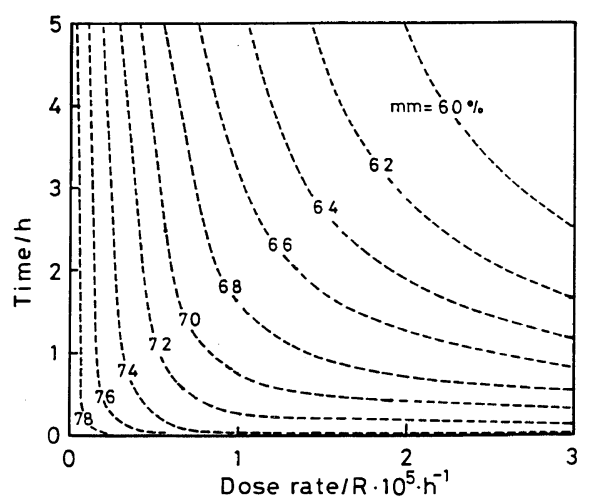

Figure 13. Experimental relations among irradiation dose rate, irradiation time and $\mathrm{mm}$ content for $\mathrm{AN}_{-}$ $n$-BM-urea system: AN-n-BM-urea $=1.0: 0.01: 3.0$. and polymer isotacticity, expressed as isotactic triad content $(\%)$ for $\mathrm{AN}-n$-BM-urea (1.0: $0.01: 3.0$, molar ratio) system. Here, aging was made at $195 \mathrm{~K}$ for 7 days. In the figure contour line is the experimental conditions under which the polymer with the same tacticity can be synthesized. Evidently, highly isotactic polymer can be readily synthesized by irradiating the complex with $\gamma$-ray at a small dose rate. Using all experimental data shown in Figure 12, the conversion, $M_{v}$ and the $\mathrm{mm}$ content were plotted against the total dose. The results are illustrated in Figures $14 a-c$. The total dose is much more fundamental quantity rather than the dose rate and the irradiation time, because the parameters such as the conversion, $M_{v}$ and the isotacticity can be expressed as single function of the total dose over wide ranges of the dose rate and the irradiation time. Similar results on the conversion and $M_{v}$ for AN-urea canal complex, in which no chain transfer reagent is added, were reported by Kawasaki et al. $^{4}$ and Yoshii et al. ${ }^{6}$ Figure 14 supports also the hypothesis presented here.

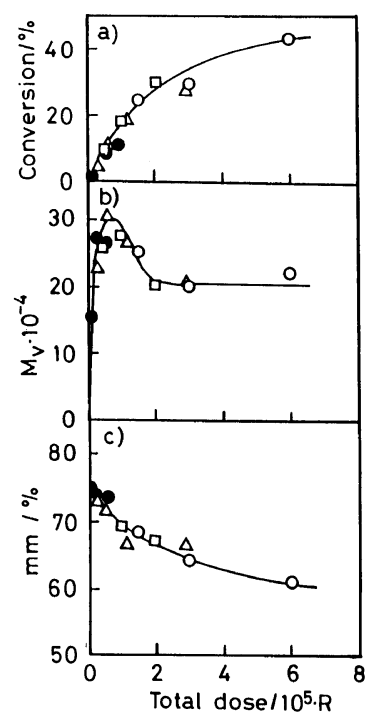

Figure 14. Effects of irradiation dose on conversion (a), $M_{v}$ (b) and $m m$ content (c) of PANs obtained in urea canal complex: $\mathrm{AN}-n$-BM-urea $=1.0: 0.01: 3.0$ (molar ratio); irradiation dose rate $0,0.30 \times 10^{5} \mathrm{R} \mathrm{h}^{-1} ; \triangle, 0.58 \times 10^{5}$ $\mathrm{Rh}^{-1} ; \square, 1.0 \times 10^{5} \mathrm{R} \mathrm{h}^{-1} ; \bigcirc, 3.0 \times 10^{5} \mathrm{R} \mathrm{h}^{-1}$. 


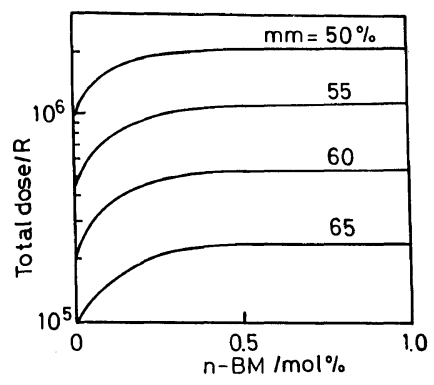

Figure 15. Experimental relations among total dose, content of $n$-BM and $m m$ content of the polymer; $\mathrm{AN}$-urea $=1.0: 1.5$ (molar ratio).

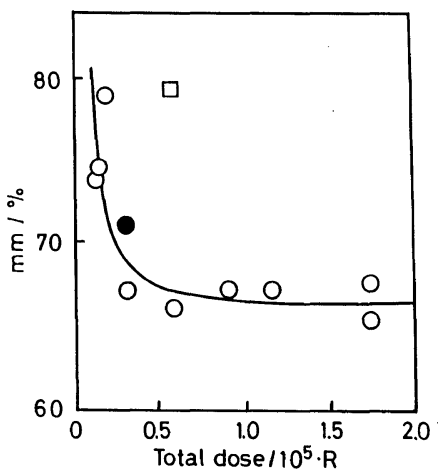

Figure 16. Relations between $\mathrm{mm}$ content and total dose: AN-urea $=1.0: 5.0$ (molar ratio); $O$, added $0.5 \mathrm{~mol} \% / \mathrm{AN}$ of $n$-BM; $\bigcirc$, without $n$-BM; $\square$, added $0.5 \mathrm{~mol} \% / \mathrm{AN}$ of $n$-BM and vaccumed at $10^{-3}$ Torr for $15 \mathrm{~h}$.

Figure 15 shows the experimental relations among the total dose, the content of $n$-BM and the $\mathrm{mm}$ content of the polymer, synthesized from $\mathrm{AN}-n$-BM-urea $(\mathrm{AN}$-urea $=1.0: 1.5$, $\mathrm{mol} / \mathrm{mol}$ ) system. In the figure contour line denotes the $m m$ content $(\%)$. In the range of $n$-BM content less than $0.5 \mathrm{~mol} \%$ (against AN) the isotacticity is functions of mercaptan content together with the total dose. As far as $n$-BM is concerned, addition of the mercaptan exceeding $0.5 \mathrm{~mol} \%$ is ineffective to increase the tacticity of polymers.

Figure 16 shows the relations between isotacticity, expressed by isotactic triad content $(\%)$, and total dose. Here, polymerization proceeded under the condition of large excess urea $(\mathrm{AN}-$ urea $=1.0: 5.0, \mathrm{~mol} / \mathrm{mol})$. In the

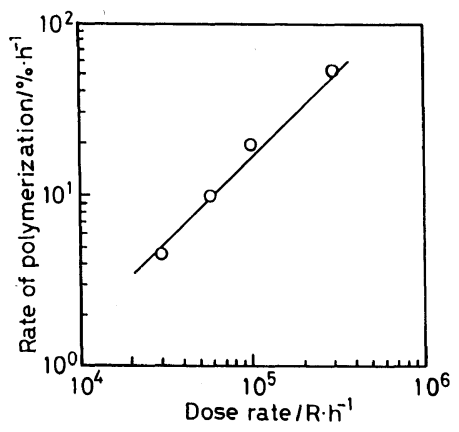

Figure 17. Dependence of dose rate on the initial rate of polymerization of $\mathrm{AN}$ in urea canal complex: $\mathrm{AN}-n$ $\mathrm{BM}$-urea $=1.0: 0.01: 3.0$ (molar ratio); irradiation temperature, $195 \mathrm{~K}$.

figure, the unfilled mark is the case where $n$-BM is added and the filled mark corresponds to the case where no chain transfer reagent is added. If all other experimental conditions are the same the isotacticity of the polymer decreases remarkably with slight increase in the total dose, approaching a constant value ( $c a .66 \%)$. By decreasing total dose, an isotacticity of $79 \%$ $\left(M_{v}=7.6 \times 10^{4}\right)$ was achieved. For comparison, the canal complex, aged at $195 \mathrm{~K}$ for 7 days, was evacuated to remove free $\mathrm{AN}$ at $10^{-3}$ Torr for $15 \mathrm{~h}$. This treatment is supposed to be effective to remove the free AN remaining in the system, but, the detailed mechanism is open for further study. The tacticity of the polymer was $79.4 \%\left(M_{v}=9.9 \times 10^{4}\right)$ and was plotted as a rectangle in Figure 16. That is, we believe, the highest isotacticity of PAN synthesized up to the present time. It is interesting to note that the highly isotactic polymer can be obtained with even a relatively larger total dose.

The rate of initial polymerization was evaluated from the relations between the conversion and irradiation time, as shown in Figure 12, and was plotted against the dose rate in Figure 17. In the case when chain transfer reagent coexisted and the dose rate was less than $3 \times 10^{5} \mathrm{Rh}^{-1}$, the rate of polymerization was in linear proportion to the dose rate, suggesting unimolecular termination reaction. It can be considered that chain 

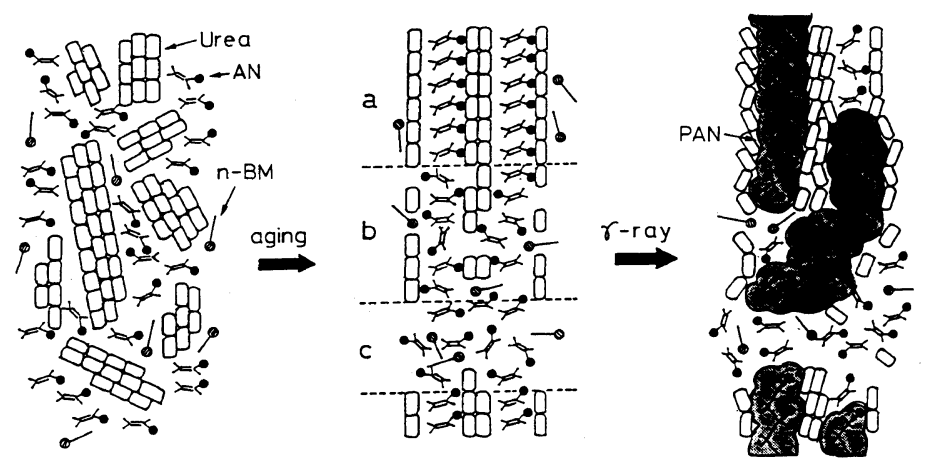

Figure 18. Schema of $\gamma$-ray irradiation polymerization of $\mathrm{AN}-n$-BM-urea canal complexes.

transfer reagent is deeply concerned with the termination reaction. When a chain transfer reagent is absent, Kawasaki et al. ${ }^{4}$ observed that the rate of initial polymerization was in linear proportion to the dose rate in the range of the dose rate from $1.60 \times 10^{3}$ to $2.24 \times 10^{4}$ $\mathrm{R} \mathrm{h}^{-1}$, Yoshii et al. ${ }^{8}$ observed that the rate of initial polymerization was proportional to the root of the dose rate when the dose rate was larger than $2.5 \times 10^{5} \mathrm{Rh}^{-1}$, suggesting the bimolecular termination reaction.

Figure 18 shows a schema of $\gamma$-ray irradiation polymerization of $\mathrm{AN}-n$ - $\mathrm{BM}$-urea canal complexes. The urea canal complex is considered to have three different morphological regions $\mathrm{a}, \mathrm{b}$ and $\mathrm{c}$, whose compositions are expected to depend on the aging period, $\mathrm{AN}$-urea ratio and amount and kind of chain transfer reagent. In region (a) an molecule is included with urea in the hexagonal crystalline form and this region has the highest activity of polymerization, which allows the complex to initiate the polymerization by a lower irradiation dose to give the polymer with the highest isotacticity. $n$-BM cannot be contaminated in this region. Region (b) is the region, in which the arrangement of $\mathrm{AN}$ monomer is a little irregular. This region is considered to be formed at half way of the complex formation or more or less by disruption due to $\gamma$-ray irradiation or by heat evolved during polymerization of AN. AN monomers in this region may polymerize only at larger irradiation dose to yield almost atactic polymers. $n$-BM is penetrable into this region and controls the polymerization in this region. A canal complex is not formed in region (c), in which AN monomer is absolutely in a free state and does not polymerize at $195 \mathrm{~K}$ even under higher irradiation dose. In addition, the PAN polymer cannot exist in the hexagonal crystal pillows with the radius of $5.2 \AA$ and the polymerization proceeds with collapse of the complex crystalline pillows.

Summarizing, in the process of polymerization of $\mathrm{AN}$ by $\gamma$-ray irradiation on $\mathrm{AN}$-urea canal complex at $195 \mathrm{~K}$, isotacticity of the polymer, as expressed by triad content $\mathrm{mm}$, increases (1) with decrease in the total dose, (2) with the addition of a chain transfer reagent and (3) with decrease in the ratio of AN-urea in the system and its maximum triad content attainable is about $80 \%$. The addition of a chain transfer reagent, especially $n$-butyl mercaptan, to the polymerization system is also quite effective to control the average molecular weight of the polymers in the adequate range, in which fibers and films can be spun or cast readily from the solutions. $n$-Butyl mercaptan is not included in the $\mathrm{AN}$-urea canal complex, suppressing the polymerization of $\mathrm{AN}$ in the less regular canal complex, allowing the polymerization of $\mathrm{AN}$ alone in the regular canal complex, which gives highly stereoregular PAN. 


\section{REFERENCES}

1. H. Clasen, Z. Elektrochem., 60, 982 (1956).

2. J. F. Brown and D. M. White, J. Am. Chem. Soc., 82, 5671 (1960).

3. D. M. White, J. Am. Chem. Soc., 82, 5678 (1960).

4. M. Kawasaki, T. Maekawa, K. Hayashi, and S. Okamura, J. Macromol. Chem., 1, 489 (1966).

5. P. Hedvig, S. Kulcár, and L. Kiss, Eur. Polym. J., 4, 601 (1968).

6. F. Yoshii, T. Abe, and O. Yoda, Kobushi Ronbunshu, 32, 399 (1975).

7. F. Yoshii, T. Abe, N. Hayakawa, and N. Tamura, Kobunshi Ronbunshu, 32, 406 (1975).

8. F. Yoshii, T. Abe, and Y. Kobayashi, Kobunshi Ronbunshu, 32, 477 (1975).

9. T. Yoshino, H. Kenjo, and K. Kuno, J. Polym. Sci., B, 5, 703 (1967).

10. K. Matsuzaki, T. Uryu, M. Okada, and H. Shiroki, J. Polym. Sci., A-1, 6, 1475 (1968).

11. K. Matsuzaki, M. Okada, and T. Uryu, J. Polym. Sci., A-1, 1, 1701 (1971).
12. K. Matsuzaki, M. Okada, and K. Goto, J. Polym. Sci., $A-1,10,823$ (1972).

13. Y. Inoue and A. Nishioka, Polym. J., 3, 149 (1972).

14. A. Nishioka, S. Ando, and Y. Inoue, Kobunshi Kagaku, 30, 139 (1973).

15. K. Kamide, H. Yamazaki, K. Okajima, and K. Hikichi, Polym. J., 17, 1233 (1985).

16. K. Kamide, H. Yamazaki, K. Okajima, and K. Hikichi, Polym. J., 17, 1291 (1985).

17. K. Kamide, H. Yamazaki, and Y. Miyazaki, Polym. J., 18, 819 (1986).

18. M. Magat, Polymer, 3, 449 (1962).

19. K. Takemoto, "Chemistry of Inclusion Compounds," Kagaku Dojin, Tokyo, 1969.

20. See for example, "Polymer Handbook 3rd Edition," J. Brandrup and E. H. Immergut, Ed., John Wiley \& Sons, New York, N.Y., 1989.

21. T. Ohmori, T. Ichikawa, and M. Iwasaki, Bull. Chem. Soc. Jpn., 46, 1383 (1973).

22. Y. Kimura, Dectoral Thesis, Tokyo University, 1974. 